\title{
DISECCIÓN ESPONTÁNEA DE ARTERIA MESENTÉRICA SUPERIOR*
}

\author{
Drs. Gabriel Figueroa B. ${ }^{1}$, Marcelo Pereira P. ${ }^{1}$, Alejandro Campos G. ${ }^{1}$, \\ Juan Pablo Moreno P. ${ }^{1}$, Als. Marcela Moraga L. ${ }^{2}$, Natalia Rivera A. ${ }^{2}$ \\ ${ }^{1}$ Departamento de Cirugía Hospital Clínico Universidad de Chile. \\ 2 Alumna de Medicina, Facultad Medicina Universidad de Chile. \\ Santiago, Chile.
}

Abstract

\section{Spontaneous dissection of superior mesenteric artery}

Introduction: Spontaneous dissection of the superior mesenteric artery (AMS) is a infrecuent pathology, representing the main frequency of isolated peripheral artery dissection, it is more common in males and occurs in the fifth decade of life. Materials and Methods: Retrospective cases analysis of patients with spontaneous dissection of AMS that were handled in our hospital, in last two years. Results: Two patients were diagnosed in this period, both active smoking and hypertensive pathology, appearing with severe abdominal and back pain. The diagnosis was made by CT angiogram of the abdomen and pelvis; establishing medical management of hypertension and standard heparin anticoagulation. Both patients had dissections at new imaging controls and one patient required exploratory laparotomy with bowel resection and intestinal anastomosis. Patients recovered satisfactorily and are in control. Conclusions: Spontaneous dissection of the SMA is a rare disease with uncertain evolution, it may occur in middle age of life, associated with smoking and hypertension. The suspected diagnosis is clinical and can be confirmed by CT angiography. Initial management remains conservative and occasionally is surgical.

Key words: Spontaneous superior mesenteric artery dissection, clinical presentation, treatment.

\section{Resumen}

Introducción: La Disección espontánea de la arteria mesentérica superior (AMS) es una patología infrecuente, representando la primera frecuencia de disección de arteria periférica aislada, ocurre más en varones en la quinta década de la vida. Material y Método: Casos en los últimos 2 años revisados en forma retrospectiva. Resultados: Dos pacientes fueron diagnosticados en este período, presentándose ambos por dolor abdominal y lumbar de gran intensidad al Servicio de Urgencia del hospital; ambos tabáquicos activos y con hipertensión arterial (HTA). El diagnóstico fue realizado por angioTC de abdomen y pelvis, instaurán-

*Recibido el 26 de agosto de 2013 y aceptado para publicación el 6 de marzo de 2014.

Los autores declaran no tener conflicto de interés.

Correspondencia: Dr. Gabriel Figueroa B.

gfigueroa@med.uchile.cl 
dose manejo médico de HTA y anticoagulación con heparina estándar. Los 2 pacientes presentaron nuevas disecciones en los controles imagenológicos y un paciente requirió laparotomía exploradora con resección intestinal y anastomosis por isquemia intestinal. Los pacientes evolucionaron satisfactoriamente y se encuentran en control. Conclusiones: La disección espontánea de la AMS es una enfermedad poco frecuente de evolución incierta, que ocurre en la edad media de la vida, asociada a tabaquismo e HTA, la sospecha es clínica y el diagnóstico por imágenes. El manejo inicial sigue siendo médico y ocasionalmente es quirúrgico.

Palabras clave: Disección espontánea arteria mesentérica superior, cuadro clínico, tratamiento.

\section{Introducción}

La disección espontánea de la arteria mesentérica superior es una patología infrecuente, con una incidencia de $0,06 \%{ }^{1}$, representando la primera frecuencia de disección de arteria periférica aislada, seguida por la carótida interna. El primer caso descrito fue en 1947, por Bauersfeld ${ }^{2}$, al cual le han seguido poco más de 100 casos en el mundo, sobre todo en la última década, debido a avances imagenológicos y angiográficos ${ }^{1}$. La disección espontánea de la AMS es más frecuente en varones (4:1), ocurriendo, en la quinta década de la vida ${ }^{3}$. Se han identificado algunos factores de riesgo, como degeneración quística de la media, displasia fibromuscular, enfermedades del tejido conectivo, arteriosclerosis, hipertensión, aneurisma de aorta abdominal y traumatismos, aunque en general ocurre en pacientes previamente sanos ${ }^{4,5}$. La presentación clínica de esta patología es dolor abdominal intenso, debido a la isquemia intestinal o hemorragia intraperitoneal por ruptura de la AMS, vómitos, diarrea, hematoquezia y distensión abdomina $1^{6-8}$. Las técnicas de imágenes modernas, como la angioTC, permiten visualizar la arteria disecada y reconstruirla tridimensionalmente, asimismo nos entregan información sobre la posible ruptura y el sufrimiento de asas intestinales, siendo el examen de elección ante la sospecha clínica ${ }^{3}$. La angiografía es utilizada en los pacientes con un empeoramiento clínico o ante la duda diagnóstica ${ }^{7,9}$. El manejo puede ser inicialmente conservador, con reposo digestivo, control de la HTA y anticoagulación ${ }^{9}$ si no existe hemoperitoneo o infarto intestinal, en este último caso se debe realizar tratamiento quirúrgico, teniendo múltiples alternativas, como la resección del segmento afectado con interposición de un injerto, reimplante de la AMS en la aorta, bypass a la arteria gastroepiplóica o trombectomía, más resección del tejido intestinal comprometi$\mathrm{do}^{1,4,6}$. Últimamente se ha sumado el manejo endovascular, con el uso de stents implantados de forma percutánea, con buenos resultados clínicos ${ }^{1,4}$. En este trabajo revisaremos los antecedentes, cuadro clínico, tratamiento y evolución de dos pacientes vistos en los últimos 2 años.

\section{Material y Método}

Revisión de las fichas clínicas, respaldos informáticos e imagenológicos, de los pacientes con diagnóstico de disección espontánea de AMS entre enero de 2012 y junio de 2013, en nuestro hospital.

\section{Resultados}

Dos pacientes fueron diagnosticados en este período:

\section{Caso 1}

Varón de 42 años, con antecedentes de HTA, tabaquismo activo y dislipidemia, que consulta a Servicio de Urgencia (SU) por cuadro de $4 \mathrm{~h}$ de dolor abdominal irradiado a flanco izquierdo, de gran intensidad (EVA 10/10). Se realiza angioTC de abdomen y pelvis, que evidencia disección de AMS (Figura 1). Se inicia manejo de HTA y terapia anticoagulante con heparina estándar. El paciente evoluciona con cefalea, por lo que requiere nuevo estudio de imágenes a las $48 \mathrm{~h}$, donde se identifican nuevas disecciones, de las arterias vertebrales, renales y tronco celíaco. Se completa el estudio etiológico, diagnosticando poliarteritis nodosa, la cual es

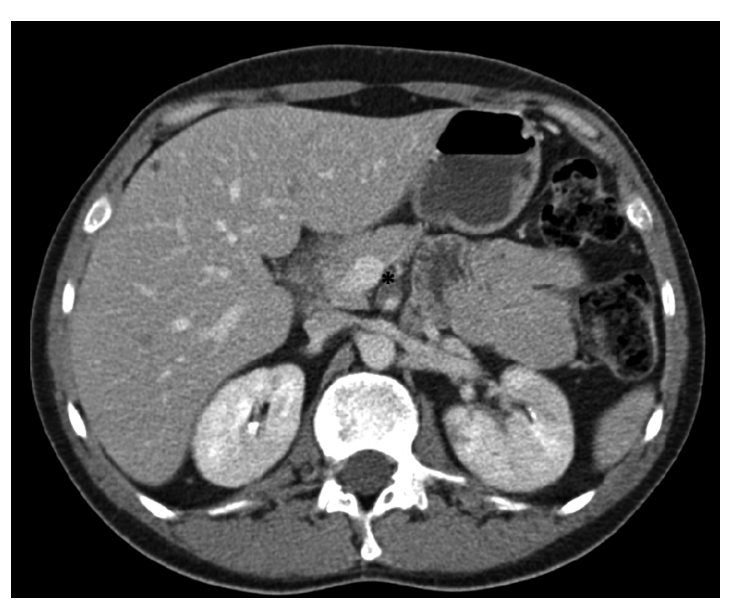

Figura 1. AngioTC abdomen y pelvis: *Disección de AMS. 
manejada con terapia esteroidal (metilprednisolona) y ciclofosfamida. El paciente evoluciona en forma satisfactoria con el manejo médico, siendo dado de alta al día 13, en buenas condiciones generales y manteniéndose en control con imágenes seriadas.

\section{Caso 2}

Mujer de 50 años, con antecedentes de HTA sin control y tabaquismo activo, que consulta a SU de $\mathrm{HCUCH}$, por cuadro de dolor abdominal difuso de gran intensidad (EVA 10/10), de 6 h de evolución. Se realiza angioTC de abdomen y pelvis, que muestra disección de AMS (Figura 2), instaurándose manejo de HTA y anticoagulación con heparina estándar. Ante la persistencia del dolor se realiza angiografía, la que confirma el diagnóstico y se optimiza el manejo médico, siendo descartado el manejo endovascular por la dificultad de acceso a las ramas yeyunales disecadas. La paciente evolucionó con aumento del dolor abdominal y deterioro clínico, requiriendo laparotomía exploradora de urgencia, la cual no mostró sufrimiento intestinal, ni sangrado activo. En el control imagenológico de las $48 \mathrm{~h}$, se evidenció hemoperitoneo masivo más neumatosis intestinal en yeyuno proximal y nuevas disecciones, en arterias ilíacas y tronco celíaco, realizando nueva laparotomía exploradora, resección de $50 \mathrm{~cm}$ de asas de yeyuno desvitalizadas y anastomosis terminoterminal manual, suspendiendo los anticoagulantes y adicionando manejo con antiagregantes plaquetarios. La paciente evoluciona satisfactoriamente, siendo dada de alta al día 24 , en buenas condiciones, encontrándose actualmente en control periódico clínico e imagenológico.

\section{Discusión}

La disección espontánea de la AMS es una enfermedad poco frecuente y de evolución incierta, que ocurre en la edad media de la vida, asociada a tabaquismo, HTA y a enfermedades reumatológicas ${ }^{1,5-9}$, la sospecha es clínica, basada en dolor abdominal de gran intensidad, el cual puede estar irradiado a zona lumbar, lo que concuerda con los casos que se presentaron en nuestro centro ${ }^{6}$. El diagnóstico es por imágenes, siendo el angioTC de abdomen y pelvis el examen de primera línea ante la sospecha clínica ${ }^{1}$. La angiografía está reservada para los casos en que se presenten dudas diagnósticas o exista un empeoramiento clínico, siendo realizada en un paciente. El manejo inicial sigue siendo médico, buscando cifras tensionales bajas y realizando anticoagulación con heparina estándar; el abordaje quirúrgico es poco frecuente en la literatura, siendo realizado principalmente para hacer resecciones intestinales,

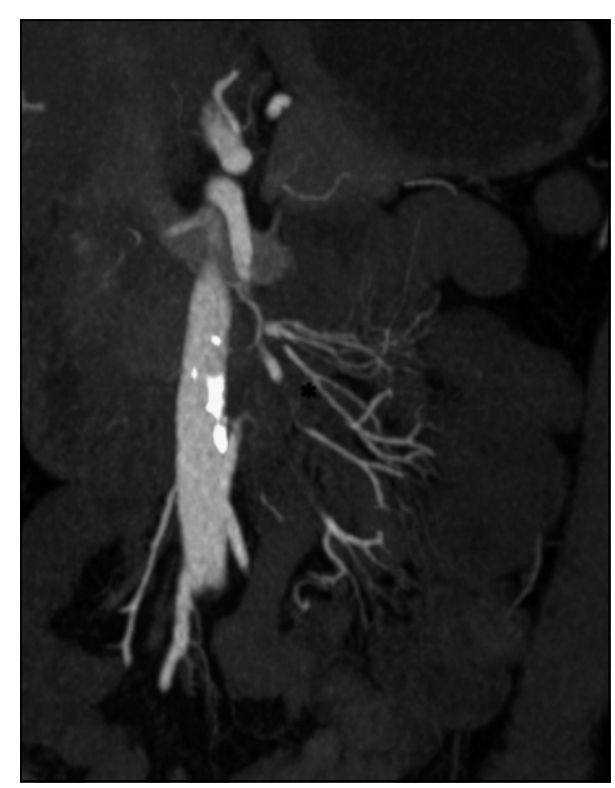

Figura 2. AngioTC abdomen y pelvis: *Disección de ramas yeyunales de AMS.

lo que puede ser necesario en forma tardía ${ }^{2,6,9}$. La evolución natural de esta patología es incierta, destacando el potencial desarrollo de nuevas disecciones arteriales, lo que nos orienta hacia la existencia de una patología sistémica, siendo necesario descartar enfermedades reumatológicas. La remisión de la sintomatología es lenta y los pacientes deben permanecer anticoagulados o con antiagregantes. La mortalidad es baja en los reportes 9 . Dada la baja frecuencia de esta patología y al diagnóstico cada vez más frecuente, debido al uso rutinario de técnicas de imágenes, en los pacientes que consultan por dolor abdominal en el SU, es necesario realizar el reporte de estos casos, para lograr definir guías de manejo clínico y documentar la evolución de los pacientes.

\section{Referencias}

1. Quintana M, Ganzarain L, Ávila C, Arruabarrena A, Fonseca J. Manejo conservador de tres casos de disección espontánea de la arteria mesentérica superior. J Angio. 2012;64:7-12.

2. Katsura M, Mototake H, Takara H, Matsushima K. Management of spontaneous isolated dissection of the superior mesenteric artery: Case report and literature review. World J Emerg Surg. 2011;6:1-6.

3. Saba L, Sanfilippo R, Atzeni M, Ribuffo D, Montisci $\mathrm{R}$, Mallarini G. Superior mesenteric artery spontaneous and isolated dissection diagnosed by using MDCTA. Eur Rev Med Pharmacol Sci. 2010;14:235-8. 
4. Baldi S, Zander T, Rabellino M, Maynar M. Endovascular management of a spontaneous dissecting aneurysm of the superior mesenteric artery: Case report and discussion of treatment options. Ann Vasc Surg. 2009;23:535-9.

5. Muse L, O’Brien A, Loyola S, Cruz F. Disección espontánea de la arteria mesentérica superior. Rev Chi Rad. 2003;8:133

6. Amabile P, Ouadissi M, Cohen S, Piquet P. Conservative treatment of spontaneous and isolated dissection of mesenteric arteries. Ann Vasc Surg. 2009;2:738-44.
7. Tameo M, Dougherty M, Calligaro K. Spontaneus dissection with rupture of the superior mesenteric artery from segmental arterial mediolysis. J Vasc Surg. 2011;53:1107-12.

8. Burgos L, Mertens R, Valdés F. Isquemia mesentérica aguda por disección espontánea y aislada de la arteria mesentérica superior. Rev Med Chile 2002;130:1282-6.

9. Zhang W, Killeen J, Chiriano J, Bianchi C, Teruya T, Abou-Zamzam A. Tratamiento de la disección aislada sintomática de arterias viscerales: ¿Es aconsejable su corrección urgente? Ann Vasc Surg. 2009;23:90-4. 